\title{
RETRACTION
}

\section{Exposure to organochlorine pesticides is independent risk factor of hepatocellular carcinoma: A case-control study}

Benhua Zhao, Heqing Shen, Feng Liu, Sheng Liu, Jianjun Niu, Fei Guo and Xueli Sun

Journal of Exposure Science and Environmental Epidemiology (2012) 22, 539; doi:10.1038/jes.2012.65

Retraction to: Journal of Exposure Science and Environmental Epidemiology (2011) 21，601-608; doi:10.1038/jes.2011.24; published online 13 July 2011

The publisher unintentionally published this earlier version of the manuscript. The correct, final version was subsequently published as Journal of Exposure Science and Environmental Epidemiology advance online publication, 14 September 2011; doi:10.1038/jes.2011.29. We regret the error. 